\title{
Prochazka, Fabian: Vertrauen in Journalismus unter Online-Bedingungen. Zum Einfluss von Personenmerkmalen, Qualitätswahrnehmungen und Nachrichtennutzung.
}

\author{
Wiesbaden: Springer VS 2020. 340 Seiten. Preis: 49,99€
}

\author{
Florian Wintterlin
}

Angenommen: 9. Dezember 2020 / Online publiziert: 18. Dezember 2020

(C) Der/die Autor(en) 2020

Vertrauen hat nicht nur in der öffentlichen Debatte um Begriffe wie Lügenpresse, sondern auch als analytische Kategorie in der Journalismusforschung in den letzten Jahren an Bedeutung gewonnen. Die Grundhypothese dahinter lautet, dass Vertrauen des Publikums für das Funktionieren des Journalismus elementar ist. Oftmals wird das mit der empirisch weitgehend unbelegten Annahme verbunden, dass Vertrauen in Medien mit dem Aufkommen des Internets und alternativen Informationsangeboten sinkt und damit die Befürchtung verknüpft, dass gesellschaftlicher Zusammenhalt bedroht ist. Prochazka widmet sich mit seiner Dissertation demzufolge einem Problemfeld, das sowohl wissenschaftlich als auch aus Sicht der Praxis als sehr relevant einzustufen ist. Er fragt danach, in welchen gesellschaftlichen Milieus Vertrauen und Misstrauen besonders verbreitet sind und was medienskeptische und medienfreundliche Personen charakterisiert. Damit leistet bereits die Fragestellung eine wichtige Differenzierung in der Diskussion über Vertrauen in Journalismus.

Im theoretischen Teil der Arbeit erläutert Prochazka zunächst die digitale Transformation von Öffentlichkeit und deren Folgen, bevor er aufbauend auf einer eigenen Vertrauensdefinition, die er von Glaubwürdigkeit und journalistischer Qualität abgrenzt, auf mögliche Einflussfaktoren auf Vertrauen in Journalismus eingeht. Im empirischen Teil der Arbeit stützt er sich auf qualitative Leitfadeninterviews mit Mediennutzer*innen und eine standardisierte Online-Befragung. Bei der Auswertung der Leitfadeninterviews setzt er einen Schwerpunkt auf das Verständnis der Befragten von Vertrauen und Journalismus sowie einen zweiten Schwerpunkt auf Qualitätswahrnehmungen des Journalismus. Damit beantwortet er vorrangig die erste Forschungsfrage, die sich auf Grundlagen von Vertrauen in Journalismus aus

\footnotetext{
Dr. F. Wintterlin $(\varangle)$

Institut für Kommunikationswissenschaft, Westfälische Wilhelms-Universität Münster, Bispinghof 9-14, 48143 Münster, Deutschland

E-Mail: florian.wintterlin@uni-muenster.de
} 
Sicht der Rezipient*innen bezieht. In der quantitativen Befragung wird auf Basis einer repräsentativen Stichprobe untersucht, inwieweit sich Zusammenhänge zwischen Personenmerkmalen, Qualitätserwartungen und -wahrnehmungen sowie Informationsnutzung mit Vertrauen in Journalismus finden lassen. Dabei wird mittels detaillierter Analysen einzelner Subgruppen des Samples versucht, Vertrauens- und Misstrauenstypen zu identifizieren.

Der Arbeit gelingt es im empirischen Teil gut, die aufgeworfenen Forschungsfragen zu beantworten. Die wesentlichen Kritikpunkte an der Arbeit betreffen demzufolge ihren theoretischen Teil. In der Definition von Vertrauen in Journalismus bezieht sich Prochazka auf psychologische Literatur und stellt dementsprechend die Akzeptanz einer Verwundbarkeit durch den Vertrauensnehmer in den Fokus. Diese Übertragung eines interpersonalen Vertrauenskonzepts auf Vertrauen in ein System wie den Journalismus ist zumindest begründungswürdig und sollte gegenüber soziologischen Betrachtungsweisen zu Systemvertrauen, die die Funktion des Systems für die Gesellschaft in den Vordergrund stellen, abgewogen werden. Eine solche Abwägung kommt im Text zu kurz. Auch die anschließende Definition von Vertrauen, verstanden als Bereitschaft, journalistische Informationen in eigene Meinungen, Einstellungen oder Verhalten zu integrieren, hätte vor dem Hintergrund der Forschung zu reliability stärker reflektiert werden können.

Die darauf folgende Literatursynopse legt Personenmerkmale, Erwartungen an Journalismus und Online-Informationsnutzung als Erklärungsfaktoren für Vertrauen in Journalismus an. Damit umfasst die Arbeit eine beeindruckende Zahl von möglichen Erklärungsansätzen aus verschiedenen Bereichen der Kommunikationswissenschaft, Psychologie und Soziologie. Auf eine Integration der Ansätze in einen gemeinsamen theoretischen Rahmen wird jedoch verzichtet. Das hat zur Folge, dass die Auswahl der Faktoren, die potenziell einflussreich für Vertrauen sein können, nicht systematisch begründet ist. Auf Ebene der einzelnen Variablenblöcke geschieht diese theoretische Argumentation dann sehr wohl und ist besonders bei den Qualitätswahrnehmungen gut gelungen. Dabei reflektiert Prochazka ausführlich theoretische und empirische Argumente und es werden an vielen Stellen alternative Interpretationen aufgezeigt.

Der empirische Teil der Arbeit ist überzeugend und stellt eine Vielzahl von interessanten Erkenntnissen dar. Die Identifizierung von Forschungsdefiziten ist schlüssig und leitet stringent zur Herleitung der Forschungsfragen über, die mit angemessenen empirischen Methoden untersucht werden. Im empirischen Teil schafft es die qualitative Studie trotz des sehr kleinen Samples, interessante Befunde zu generieren und kann als Vorstudie für die quantitative Studie eingeordnet werden, die konkrete Hypothesen für die anschließende Überprüfung generiert. Das entwickelte Messinstrument der quantitativen Studie sticht unter den vorhandenen Messinstrumenten für Vertrauen in Journalismus deutlich hervor, weil es mit der Akzeptanz von Risiko über zukünftige Entwicklungen einen wesentlichen Kern des Vertrauens erfasst, der in anderen Messinstrumenten, die nur retrospektiv nach der Erfüllung von Erwartungen fragen und nicht in die Zukunft gerichtet sind, fehlt. Zusätzliche Angaben zur Skalenentwicklung (Itemgenerierung, Tests an anderen Samples, konfirmatorische Analysen) wären jedoch hilfreich gewesen. In Bezug auf journalistische Qualität ist die Differenzierung von Qualitätserwartungen nach ihrer wahrgenommenen Wich- 
tigkeit sowie der Soll-Ist-Vergleich eine wichtige Ergänzung der vorhandenen Literatur. Der Detailgrad der Auswertung überzeugt und führt zu Erkenntnissen, die eine differenzierte Betrachtung von Zusammenhängen zwischen den Variablen zulassen.

Insgesamt leistet die Arbeit auf empirischer Ebene eine detaillierte Analyse mit hoher Tiefe, die einige interessante Ergebnisse in Bezug auf Antezedenzien von Vertrauen in Journalismus liefert, besonders mit Blick auf die interne Diversität von Medienskeptikern bzw. Journalismus-Fans. Diese Ergebnisse machen die Arbeit sowohl für die Praxis als auch für die Wissenschaft in höchstem Maße anschlussfähig.

Funding Open Access funding enabled and organized by Projekt DEAL.

Open Access Dieser Artikel wird unter der Creative Commons Namensnennung 4.0 International Lizenz veröffentlicht, welche die Nutzung, Vervielfältigung, Bearbeitung, Verbreitung und Wiedergabe in jeglichem Medium und Format erlaubt, sofern Sie den/die ursprünglichen Autor(en) und die Quelle ordnungsgemäß nennen, einen Link zur Creative Commons Lizenz beifügen und angeben, ob Änderungen vorgenommen wurden.

Die in diesem Artikel enthaltenen Bilder und sonstiges Drittmaterial unterliegen ebenfalls der genannten Creative Commons Lizenz, sofern sich aus der Abbildungslegende nichts anderes ergibt. Sofern das betreffende Material nicht unter der genannten Creative Commons Lizenz steht und die betreffende Handlung nicht nach gesetzlichen Vorschriften erlaubt ist, ist für die oben aufgeführten Weiterverwendungen des Materials die Einwilligung des jeweiligen Rechteinhabers einzuholen.

Weitere Details zur Lizenz entnehmen Sie bitte der Lizenzinformation auf http://creativecommons.org/ licenses/by/4.0/deed.de.

Dr. Florian Wintterlin ist wissenschaftlicher Mitarbeiter am Institut für Kommunikationswissenschaft der Universität Münster. 